\title{
THC:CBD in Daily Practice: Available Data from UK, Germany and Spain
}

\author{
Óscar Fernández
}

Institute of Clinical Neuroscience, Neurology Department, Hospital Regional Universitario Carlos Haya, FIMABIS, Malaga, Spain

\author{
Key Words \\ Multiple sclerosis spasticity · Patient registry $\cdot$ Safety \\ THC:CBD oromucosal spray
}

Conclusions: The homogeneity between these observational studies supports the interest in THC:CBD oromucosal spray for management of MS spasticity in daily practice.

(c) 2016 S. Karger AG, Basel

\section{Introduction}

In June 2010, health authorities in the United Kingdom (UK) and Spain granted marketing approval to Sativex ${ }^{\circledR}$ (THC:CBD oromucosal spray) based on the results of a comprehensive development plan of clinical trials. As per its approved European label, THC:CBD oromucosal spray is indicated for symptom improvement in adult patients with moderate to severe multiple sclerosis (MS) spasticity, who have not responded adequately to other anti-spasticity medication and who demonstrate clinically significant improvement in spasticityrelated symptoms during an initial trial of therapy [1]. Marketing approval has since been granted in a growing number of European Union countries.

Whereas randomized controlled trials (RCTs) are the gold standard for evidence generation and comparison of treatment options, data collected in daily practice complement the results of experimental studies by providing information from larger samples and under prescribed conditions use, outside the extraordinary selection criteria of clinical trials, without randomized allocation or placebo control groups and tracked under usual conditions.

\section{KARGER}

E-Mail karger@karger.com

www.karger.com/ene (c) 2016 S. Karger AG, Base

0014-3022/16/0757-0001\$39.50/0
Professor Óscar Fernandez

Institute of Clinical Neuroscience, Neurology Department, IBIMA

(Instituto de Investigación Biomédica de Málaga), Hospital Universitario Regional de

Málaga - Universidad de Málaga Avda, de Carlos Haya sn, ES-29010 Málaga (Spain)

E-Mail oscar.fernandez.sspa@juntadeandalucia.es 
Naturalistic setting studies enrich clinical trial results by providing effectiveness rates, validating safety and tolerability profiles and describing possible adjustment of dose ranges. Over the past 5 years, several large post-approval observational studies have investigated the effectiveness and safety of THC:CBD oromucosal spray under use in everyday clinical practice. In this review, key findings of post-approval studies with a focus on safety are examined and compared.

\section{UK/German/Swiss THC:CBD Risk Management Plan}

Usual European Medicines Agency processes when applying for approval of a new medicine require the submission of a risk management plan. As part of this framework, an observational patient registry was set up in 2010 in the UK to collect safety data on THC:CBD oromucosal spray and was followed by the establishment of similar registries in Germany and Switzerland. The primary focus of the risk management plan was to determine the long-term safety profile of THC:CBD oromucosal spray under usual clinical practice conditions. The initial target sample was 3,000 patients.

The UK/German/Swiss THC:CBD risk management plan involves voluntary retrospective reporting. All prescribers of THC:CBD oromucosal spray in the UK and those from about 30 selected MS centres in Germany and Switzerland are requested to complete a 6-monthly Case Record Form about patients' use of the spray and adverse events for all patients who have received at least one prescription. To identify potential adverse events of special interest with a cannabis-based medicine, targeted questions are asked regarding addiction potential, abuse/misuse, long term psychiatric effects, memory impairment, driving ability and fall events requiring medical attention.

Two interim analyses of the registry were previously reported $[2,3]$. A more recent report has been prepared subsequent to closure of the main registry in the UK upon regulatory agency advice that sufficient data were available from UK patients to characterize the long-term safety profile of THC:CBD oromucosal spray. The registries in Germany and Switzerland remain open.

The analysis reports on 941 patients (761 from the UK, 178 from Germany, 2 from Switzerland) whose data on use of THC:CBD oromucosal spray were collected via the registry up to February 2015 [4]. Reported patients in the UK represented $22 \%$ of the 3,493 patients who had been prescribed THC:CBD oromucosal spray in that country since June 2010 . The cohort was $57 \%$ female with a mean
Table 1. Tolerability to THC:CBD oromucosal spray in the UK/ German/Swiss risk management plan [4]

\begin{tabular}{ll}
\hline Most common adverse events & Incidence, $\%(\mathrm{n}=941)$ \\
\hline Psychiatric adverse events & 6 (including depression, 3) \\
Falls & 6 \\
Dizziness & 3 \\
Fatigue & 2 \\
Suicidality & 2 \\
Driving worsening/improvement & $2 / 7$
\end{tabular}

age of $51 \pm 10.8$ years. Long-term continuation rates after a mean follow-up time of about 1 year were $68 \%$. Among patients who had discontinued treatment, about one-third cited lack of effectiveness and about one-quarter cited lack of tolerability/adverse events. The mean recorded dose of THC:CBD oromucosal spray was $5.4 \pm 4.9$ sprays/day.

With respect to adverse events of special interest, MSrelated psychiatric events of significance (including depression) and falls requiring medical attention were each reported in $6 \%$ of patients and suicidality in $2 \%$ of patients (table 1). There was no evidence of addiction, abuse/misuse or memory impairment. The proportion of patients reporting improvement in driving ability exceeded that reporting impairment (7 vs. $2 \%$ ), likely reflecting the symptomatic improvement in spasticity. The other commonly reported adverse events of dizziness and fatigue are within the known safety profile of THC:CBD oromucosal spray [1].

The data from this large registry covering 2,200 patient-years of exposure to THC:CBD oromucosal spray support its positive benefit-risk balance during longterm use in clinical practice for the treatment of resistant MS spasticity. A relevant proportion of patients gained sufficient benefit to warrant continued treatment at doses lower than those used in RCTs, and no new safety signals were identified.

\section{Spanish Safety Study}

In Spain, health authorities supported the collection of safety data on THC:CBD oromucosal spray at the time of its approval in 2010. The Spanish safety study involved 207 patients from 13 specialized MS centres across Spain who were prescribed THC:CBD oromucosal spray as add-on therapy for moderate to severe treatment-resistant MS spasticity between July 2011 and December 2012. In contrast to the UK/German/Swiss risk management plan, patients in Spain were followed prospectively and 
the focus was on treatment continuation as well as on safety. Outcomes were reported at 6 and 12 months. Final data are now available [5].

The sample of 204 evaluable patients was $62 \%$ female, with a mean age of $48.6 \pm 9.7$ years. After 6 months' exposure to THC:CBD oromucosal spray, treating physicians considered that 143 patients $(70.1 \%)$ were deriving sufficient benefit to continue with treatment. After 12 months' exposure, the corresponding figure was 132 patients (64.7\%). The mean dose of THC:CBD oromucosal spray at both evaluation time points was 6.6 sprays/day.

Approximately, one-third of patients (35\%) discontinued THC:CBD oromucosal spray permanently during the observation period, $30 \%$ prior to 6 months' exposure and 5\% between 6 and 12 months' exposure. The most common reasons for treatment discontinuation were lack of tolerability and/or lack of effectiveness in roughly equal proportions during the first 6 months and mainly lack of tolerability among the 9 patients who discontinued treatment between 6 and 12 months.

A total of 57 adverse events of mainly mild or moderate intensity were reported in 41 patients ( $20 \%$ of the cohort). Incidences of adverse events of special interest were low (table 2). Among 57 still-driving patients with 12 months' exposure to THC:CBD oromucosal spray, 2 reported improvement and 55 reported no change in their ability to drive.

\section{Conclusions}

Despite methodological differences between the UK/ German/Swiss registry study and Spanish safety study, similar key data were collected in each study, and the findings were highly consistent. After about 1 year's treatment with THC:CBD oromucosal spray, the majority of patients in both studies were deriving sufficient benefit in
Table 2. Tolerability to THC:CBD oromucosal spray in the Spanish safety study [5]

\begin{tabular}{lc}
\hline Adverse events & $\begin{array}{l}\text { Incidence, } \mathrm{n}(\%) \\
(\mathrm{n}=204)\end{array}$ \\
\hline Any adverse event & $41(20.0)$ \\
Significant psychiatric or psychotic event & $5(2.4)^{*}$ \\
Reduced driving ability & $1(0.5)$ \\
Falls requiring medical attention & $1(0.5)$ \\
Suicidal thoughts/attempted suicide & $0(0)$ \\
Abuse/misuse & $0(0)$ \\
Other & $27(16.8)$ \\
\hline
\end{tabular}

* Four cases of mild depressive syndrome, 1 case of moderate psychotropic effects.

the opinion of treating physicians to warrant continued use (68\% in the registry study; $64.7 \%$ in the Spanish safety study). Mean daily doses of THC:CBD oromucosal spray in each study (5.4 and 6.6 sprays/day, respectively) were lower than the 8.3 sprays/day reported in a phase III enriched-design RCT [6]. Incidences of adverse events of special interest with a cannabis-based medicine were low, and there were no signals to indicate abuse, diversion or dependence. Collectively, the studies provide evidence of sustained benefit and good safety with real-world use of THC:CBD oromucosal spray in patients with treatmentresistant MS-related spasticity and are highly relevant to clinicians for application in everyday practice.

\section{Disclosure Statement}

Professor Ó. Fernández has received honoraria as a consultant in advisory boards and as chairman or lecturer in meetings, and has also participated in clinical trials and other research projects promoted by Actelion, Allergan, Almirall, Bayer Schering, BiogenIdec, Genzyme, Merck-Serono, Novartis, Roche and/or Teva.

\section{References}

1 Sativex Oromucosal Spray: Summary of Product Characteristics. 2015. https://www. medicines.org.uk/emc/medicine/23262 (accessed November 2015).

2 Wright S, Etges T: An observational post approval registry study of patients prescribed Sativex ${ }^{\circledR}$. Results from clinical practice. Mult Scler 2012;18:S30.

3 Eltayb A, Etges T, Wright S: An observational post approval registry study of patients prescribed Sativex ${ }^{\circledR}$. Results from clinical practice. Mult Scler 2013;19(suppl 1):480.
4 Etges T, Taylor A, Karolia K, Lauder H, Daka $B$, Wright S: A final report of an observational post-marketing safety registry of patients primarily from the United Kingdom who have been prescribed an oromucosal spray containing $\Delta 9$-tetrahydrocannabinol and cannabidiol (THC:CBD). Mult Scler 2015; 21(suppl 11):610.

5 Oreja-Guevara C: Observational safety study of THC: CBD oromucosal spray (Sativex) in multiple sclerosis patients with spasticity. Clin Exp Pharmacol 2015;5:184.
6 Novotna A, Mares J, Ratcliffe S, Novakova I, Vachova M, Zapletalova O, Gasperini C, Pozzilli C, Cefaro L, Comi G, Rossi P, Ambler Z, Stelmasiak Z, Erdmann A, Montalban X, Klimek A, Davies P; Sativex Spasticity Study Group: A randomized, double-blind, placebo-controlled, parallel-group, enriched-design study of nabiximols* (Sativex $\left({ }^{\circledR}\right)$ ), as add-on therapy, in subjects with refractory spasticity caused by multiple sclerosis. Eur J Neurol 2011;18:1122-1131. 